ISSN 0975.3303

Mapana J Sci, 9, I (2010), $31-40$

https:/ / doi.org/ 10.12725/ mjs.16.4

\title{
ASSOCIATE RING GRAPHS
}

\author{
M. James Subhakar*
}

\section{ABSTRACT}

$R$ is a commutative ring with unity. The associate ring graph $A G(R)$ is the graph with the vertex set $V=R-\{0\}$ and edge set $E=\{(a, b) / a, b$ are associates and $a \neq b\}$. Since the relation of being associate is an equivalence relation, this graph is an undirected graph and also each component is complete. In this paper, I present some of the interesting results majority of which are for the ring of integers modulo $n, n$ is a positive infeger.

1) $A G(R)$ is an emply graph if $R$ is a Boolean ring.

2) $A G(Z)$ is complete if and only if $n$ is prime.

3) If $n$ is even then $A G\left(Z_{n}\right)$ has an isolated vertex $n / 2$.

4) If $p$ is prime and $p \neq 2$, then $A G\left(Z_{2 p}\right)=K, \cup K_{\rho, 1} \cup K_{\rho-1}$.

5) $A G\left(Z_{p 2}\right)=K_{p-1} \cup K_{p(p-1)}$

6) $A G\left(Z_{p q}\right)=K_{p-1} \cup K_{q, 1} \cup K_{p q-p \sim q+1}$

7) $A C$-program to find the components of $A G\left(Z_{n}\right)$.

\section{Introduction}

The motivation for associate ring graphs is from zero-divisor graphs defined by I.Beck in the year 1988. He introduced the idea of these graphs for commutative rings $R$ with unity 1 . He defined $\Gamma_{0}(R)$ to be the graph whose vertices are elements of $R$ and in which two vertices $x$ and $y$ are adjacent if and only if $x y=0$. Beck was

* Department of Mathematics, Noble College, Machilipatnam, A.P.521001. misubhakar@gmail.com (Corresponding author) 
mostly concerned with coloring $\Gamma_{0}(R)$. In his paper [ 1$]$ he studied the subgraph $\Gamma(R)$ whose set of vertices is $Z(R)^{\circ}=Z(R)-\{0\}$ where $Z(R)$ is the set of zero-divisors of $R . \Gamma(R)$ is non empty unless $R$ is an integral domain and, by a result of G. Ganesan, $Z(R)$ and hence $(R)$ is finite if and only if $R$ is finite. It is shown that $\Gamma(R)$ is connected with diam $(\Gamma(R)) \mathrm{d} \Omega$. Lot of results were subsequently developed (Some of them can be seen in [2] and [3]) by several authors for zero-divisor graphs. If $R$ is a field then $(R)$ is empty or $(R)$ has no edges when all non-zero elements are used as vertices. Since a field is very rich with respect to algebraic structure, it is quite reasonable to associate a graph which is also rich graph theoretically. We know that complete graphs take this place. So I thought of defining a graph from a ring $R$ so that it is complete when $R$ is a field. This graph is nothing but the so called ASSOCIATE RING GRAPH.

\section{Preliminaries}

All the fundamental concepts of ALGEBRA are from [4] and of GRAPH THEORY are from [5].

\section{Associate Ring Graphs}

3.1 Associate ring graph: Let $R$ be a ring with unity 1 (not necessarily commutative). The associate ring graph of $R$ denoted by $A G(R)$ is the graph $(V, E)$ where the vertex set $V=R-\{0\}$ and the edge set $E=\{(a, b) / a$ is an associate of $b$ and $a \neq b\}$.

Note: Throughout this paper a ring always means a ring with unity 1 .

3.2 Orbit of an element of a ring: If $a$ is an element of a ring $R$ then the orbit of $a$ denoted by $\operatorname{Or}(a)$ is defined as $\operatorname{Or}(a)=\{a . u \mid v$ is a unit in $R\}$.

3.3 Theorem: The orbits of elements of a ring are either identical or disjoint.

Proof: Let $R$ be a ring and $a, b$ are two elements of $R$.

If $\operatorname{Or}(a)$ and $\operatorname{Or}(b)$ are disjoint we have nothing to prove.

Suppose that $\operatorname{Or}(a) \cap \operatorname{Or}(b) \neq \varnothing$.

Let $c \in \operatorname{Or}(a) \cap \operatorname{Or}(b)$. Then $c=a . v$ and $c=b . v$ for some units $u, v$ in $R$.

$\therefore a \cdot u=b \cdot v \Rightarrow a=b \cdot\left(v \cdot v^{-1}\right)$ and $b=a \cdot\left(u \cdot v^{-1}\right)$ and so $a$ and $b$ are

associates.

Let $x$ be an arbitrary element in $\operatorname{Or}(a)$. Then $x=a . s, s$ is a unit in $R$.

So $x=b \cdot\left(v \cdot u^{-3}\right) \cdot s$

i.e., $x=b .\left(v \cdot u^{-1} . s\right)$ 
i.e., $x=b$. ( $a$ unit in R).

i.e., $x \in \operatorname{Or}(b)$ and so $\operatorname{Or}(a) \subseteq \operatorname{Or}(b)$. Similarly we can show that $\operatorname{Or}(b) \subseteq$ $\operatorname{Or}(a)$.

Thus Or(a) $=$ Or(b).

Hence the Orbits of any two elements of a ring are either disjoint or identical.

3.4 Observation: Since the relation of being associative is an equivalence relation it partitions $R$ in to disjoint sets and it can be easily seen that the equivalence class containing an element $a$ is nothing but $\operatorname{Or}(a)$. Thus our graph contains connected components equal in number to the number of disjoint equivalence classes except $\{0\}$.

3.5 Example 1. Consider the ring $(Z,+, \cdot)$ of integers. We know that 1 and -1 are the only units of $Z$. Therefore for any $O \neq a$ in $Z, \operatorname{Or}(a)=\{a,-a\}$. Hence $A G(Z)$ consists of infinite number of components each is $a K_{2}$.

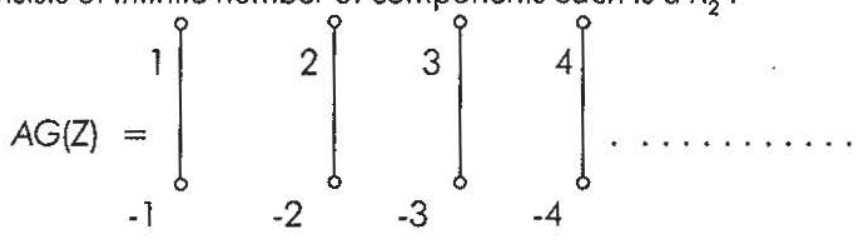

Therefore $A G(Z)=K_{2} \cup K_{2} \cup K_{2} K_{2} \ldots \ldots \ldots$

Example 2. Consider $\left(Z_{5},+_{5}, X_{5}\right)$. This is a field. Every non zero element is a unit and so any two non-zero elements are associates. Hence the graph is a complete graph with four vertices $1,2,3,4$.

The graph $A G\left(Z_{s}\right)$ is

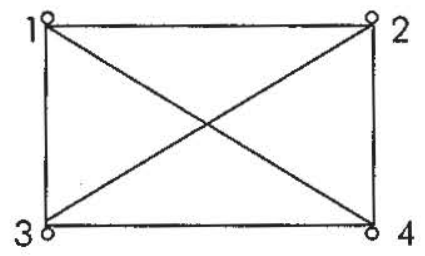

Hence $\quad A G\left(Z_{5}\right)=K_{4}$.

Example 3. Consider $\left(Z_{6},{ }_{6}, x_{6}\right)$. Here $Z_{6}=\{0,1,2,3,4,5\}$.

The Units of $Z_{6}$ are 1,5 .

$\operatorname{Or}(1)=\{1 \times 6,1 \times 6\}=\{1,5\}$. 
$\operatorname{Or}(2)=\{2,4\}$

$\operatorname{Or}(3)=\{3\}$

$\operatorname{Or}(4)=\{2,4\}$

$\operatorname{Or}(5)=\{1,5\}$.

The graph $A G\left(Z_{6}\right)$ is

$$
{ }_{5}^{19}
$$$$
{ }_{4}^{40}
$$

Hence $A G\left(Z_{6}\right)=K_{1} \cup K_{2} \cup K_{2}$.

3.6 Theorem: $A G(R)$ is an empty graph (without edges) if $R$ is a Boolean ring.

Proof: Let $R$ be a Boolean ring with unity 1 .

We show that $R$ has no units other than the unity $l$.

Let $0 \neq a$ be $a$ unit in $R$. i.e., $a . b=1$ for some $0 \neq b$ in $R$. Since $R$ is Boolean, $a^{2}=a$.

Now $a \cdot b=1 \Rightarrow a \cdot(a \cdot b)=a \cdot 1 \Rightarrow a^{2} \cdot b=a \Rightarrow \cdot b=a \Rightarrow=a$.

Hence 1 is the only unit in $R$. Therefore the orbit of every non-zero element of $R$ contains only itself.

Hence $A G(R)$ has no edges.

3.7 Theorem: $A G\left(Z_{n}\right)=K_{n-1}$ (the complete graph with $n-$ - vertices) if and only if $n$ is prime.

Proof: Suppose that $A G\left(Z_{n}\right)$ is complete.

i.e., every pair of non zero elements of $Z_{n}$ are connected by an edge.

We know that $Z_{n}$ is a commutative ring with unity 1 .

If $a$ is any non-zero element of $Z_{n}$ then $a$ and 1 are joined by an edge. i.e., $a$ and 1 are associates. 
i.e., $1=u$. a for some unit $u$ in $Z_{n}$.

i.e., $\alpha$ is an invertible element in $Z_{n}$.

i.e., every non zero element in $Z_{n}$ is invertible.

Thus $Z_{n}$ is a field and hence $n$ is prime.

Conversely suppose that $n$ is prime.

Therefore $Z_{n}$ is a field.

Let $x$ and $y$ be two non zero elements of $Z_{n}$.

Since $Z_{n}$ is a field $x$ and $y$ are units.

So $x^{-1} \cdot y$ is also a unit in $Z_{n}$.

We have $x \cdot\left(x^{-1} \cdot y\right)=y$.

$\Rightarrow x$ is an associate of $y$.

$\Rightarrow x$ and $y$ are joined by an edge.

Thus every pair of non-zero elements of $Z_{n}$ are joined by an edge.

Hence $A G\left(Z_{n}\right)$ is complete.

3.8 Theorem: If $n$ is even then $A G\left(Z_{n}\right)$ has an isolated vertex namely $n / 2$.

Proof: Suppose $n$ is even.

i.e., $n=2 m$ for some $m$ in $N=\{1,2,3, \ldots .$.$\} .$

We show that $m=n / 2$ is an isolated vertex in $A G\left(Z_{n}\right)$.

We know that the units of $Z_{n}$ are the non-zero elements of $Z_{n}$ which are relatively prime to $n$. Since $n$ is even these units must be odd.

Let $a=2 k+1$ be a unit in $Z_{n}$.

Then we have $m . a=m \cdot(2 k+1)=2 m k+m=n k+m=m$ (Since $n k=0$ in $Z_{n}$ ).

Thus the only associate of $m$ is $m$ itself.

Since $A G\left(Z_{n}\right)$ has no self loops $m$ is an isolated vertex of $A G\left({ }_{n}\right)$. 
3.9 Theorem: If $n=2 p$ where $p$ is a prime $(\neq 2)$ then $A G\left(Z_{n}\right)=K_{1} \cup K_{p .1} \cup K_{p .1}$. Proof: Let $n=2 p$. By 3.8, $A G\left(Z_{n}\right)$ has an isolated vertex $n / 2=p$. So $A G\left(Z_{n}\right)$ contains $K_{1}$. Also $A G\left(Z_{n}\right)$ has a component $K_{\phi(n)}=K_{\varphi(2 p)}=K_{\phi(2) \varphi(p)}$ $=K_{p .1} \cdot$

It is enough to prove that the graph has only one component left and that is also a $K_{p-1}$.

We show that the remaining vertices other than $p$ and the units in $K_{\phi(n)}=K_{p \cdot 3}$ forms the vertices of the other $K_{p .1}$.

Clearly the number of vertices remaining are $[(n-1)-(p-1)-1]=p-1$.

We have $m$ is a unit if and only if $(m, 2 p)=1$.

If and only if $m$ is odd and not a multiple of $p$.

If and only if $m$ is odd and $m \neq p$.

If and only if $m=1,3,5, \ldots \ldots,(p-2),(p+2), \ldots \ldots,(2 p-1)$.

Therefore the set of remaining elements is $D=\{2,4, \ldots .(p-1),(p+1), \ldots$ $(2 \mathrm{p}-2)\}$.

We show that the orbit of any general element $2 k$ of $D$ is $D$. The associates of $2 k$ are $2 k(1), 2 k(3), \ldots \ldots \ldots \ldots, 2 k(p-2), 2 k(p+2), \ldots \ldots, 2 k(2 p-1)$. These products are all even and so are elements of $D$. We show that that these products are distinct.

Suppose that $2 k(2 m-1)=2 k(2 s-1)$ where $m \neq s$ and $m>s$.

So $2 p$ divides $2 k(2 m-1)-2 k(2 s-1)=4 k(m-s)$.

So $p$ divides $2 k(m-s)$.

Since $p$ does not divide 2 and $k$, we must have $p \mid(m-s)$.

Since $(m-s)<p$ we must have $m=s$, a contradiction.

Thus the orbit of $2 k$ is $D$. Therefore every element of $D$ is an associate to every other element of $D$. This shows that the elements in $D$ form the required $K_{\rho-1}$.

Hence $A G\left(Z_{2 \rho}\right)=K_{1} \cup K_{\rho, 1} \cup K_{\rho, 1}$. 
3.10 Theorem: $A G\left(Z_{\rho 2}\right)=K_{p .1} \cup K_{p(p-1)}$.

Proof: Let $p$ be a prime number.

We have $Z_{\rho 2}=\left\{0,1,2, \ldots,\left(p^{2}-1\right)\right\}$.

For any $0 \neq a$ in $Z_{\rho 2},\left(a, p^{2}\right)=1$ if and only if $p$ does not divide $a$.

if and only if $a$ is not a multiple of $p$.

Hence $\operatorname{Or}(1)=$ units of $Z_{p 2}=\{1,2, \ldots,(p-1),(p+1), \ldots(2 p-1),(2 p+1), \ldots$ $\left.(p-1) p-1,(p-1) p+1, \ldots\left(p^{2}-1\right)\right\}$.

The remaining non-zero elements of $Z_{p 2}$ are $p, 2 p, 3 p, \ldots(p-1) p$.

Obviously the number of elements in $\operatorname{Or}(1)=$ number of units $=\left(p^{2}-1\right)-$ $(p-1)=p(p-1)$.

Thus $A G\left({ }_{p 2}\right)$ has $K_{\rho(0.1)}$ as a component.

To prove the theorem it is enough to show that the remaining $(p-1)$ nonunits(zero-divisors) forms a $K_{\rho-1}$.

Let $D=\{p, 2 p, \ldots,(p-1) p\}$.

We have $\operatorname{Or}(p)=\{p .1, p .2, \ldots, p \cdot(p-1), p \cdot(p+1), \ldots \ldots\}$.

Clearly the first $(p-1)$ elements of $\operatorname{Or}(p)$ are elements of $D$.

So $D$ is a subset of $\operatorname{Or}(p)$.

Since $p$ is a non-unit, all elements of Or(p) are non-units.

So $\operatorname{Or}(p) \cap \operatorname{Or}(1)=\varnothing$

Therefore $\operatorname{Or}(p)$ is a subset of $\{\operatorname{Or}(1)\}^{c}=D$.

From (1) and (2) we get $\operatorname{Or}(p)=D$.

Thus the elements $p, 2 p, 3 p, \ldots,(p-1) p$ of $\operatorname{Or}(p)$ forms the vertices of the required $K_{(p-1)}$.

Hence $A G\left(Z_{p 2}\right)=K_{p .1} \cup K_{p(0.1)}$.

3.11 Theorem: $A G\left(Z_{p q}\right)=K_{(p \cdot 1)} \cup K_{(q \cdot 1)} \cup K_{p q \cdot p \cdot q+1}$.

Proof: Without loss of generality we assume that $p<q$. The cases when $p=2$ and $p=q$ are already dealt in 3.9 and 3.10 respectively. 
Now $n$ is a unit in $Z_{p q}$ if and only if $(n, p q)=1$.

If and only if $n$ is neither a multiple of $p$ nor a multiple of $q$.

Also $n$ is not a unit if and only if $n$ is either a multiple of $p$ or a multiple of $q$.

We have $\operatorname{Or}(1)=\{1,2, \ldots,(p-1),(p+1), \ldots(q-1),(q+1), \ldots \ldots,(p q-1)\}$.

Obviously $n[\operatorname{Or}(1)]=i(p q)=i(p) \cdot j(p)=(p-1) \cdot(q-1)=p q-p-q+1$.

Thus $K_{p q \cdot p-q+3}$ is a component of $A G\left(Z_{p q}\right)$.

Since $p, q$ are distinct primes they are not associates.

For let $p=u \cdot q$ where $u$ is $a$ unit in $Z_{p q}$.

i.e., $p-u . q$ is divisible by $p q$.

i.e., $p-u \cdot q=k . p q$ where $k$ is an integer.

i.e., $p=q(u+k p)$.

i.e., $p$ is divisible by $q$, a contradiction.

Hence $\operatorname{Or}(p) \cap \operatorname{Or}(q)=\varnothing$.

Here $(p+q)$ is neither a multiple of $p$ nor a multiple of $q$.

So $(p+q)$ is a unit in $Z_{p q}$ and hence $p(p+q)$ is an associate of $p$.

But $p(p+q)=p^{2}+p q=p^{2}\left(\right.$ since $p q=0$ in $\left.Z_{p q}\right)$.

Thus $p^{2}$ is an associate of $p$.

Similarly we can show that $p^{3}, p^{4}, \ldots$ are associates of $p$.

Thus 1.p, 2.p , ... p.p , ...., (q-1).p are distinct elements in Or(p).

Therefore $n[\operatorname{Or}(p)] \geq q-1$ and similarly $n[\operatorname{Or}(q)] \geq p-1$.

We have Or(I) Or(p) Or $(q) \subseteq Z_{\rho q} \cdot$

Also $n[\operatorname{Or}(1) \operatorname{Or}(p) \operatorname{Or}(q)]=n[\operatorname{Or}(1)]+n[\operatorname{Or}(p)]+n[\operatorname{Or}(q)]$ (the union is disjoint)

$$
\begin{aligned}
& \geq(p q-p-q+1)+(p-1)+(q-1) \\
& =p q-1 \\
& =n\left[Z_{p q}\right]
\end{aligned}
$$


Therefore $n[\operatorname{Or}(1) \cup \operatorname{Or}(p) \cup \operatorname{Or}(q)] \geq n\left[Z_{p q}\right]$

From (1) and (2) we get $\operatorname{Or}(1) \cup \operatorname{Or}(p) \cup \operatorname{Or}(q)=Z_{p q}$.

Now Or $(p)$ cannot contain more than $(q-1)$ elements otherwise $\operatorname{Or}(q)$ contains less than $(p-1)$ elements which is not true. Thus $n[\operatorname{Or}(p)]=(q-1)$ and so $n[\operatorname{Or}(q)]=(p-1)$.

Hence $Z_{p q}$ has only three distinct orbits namely $\operatorname{Or}(1), \operatorname{Or}(p)$ and $\operatorname{Or}(q)$ with elements $(p q-p-q+1),(q-1)$ and $(p-1)$ respectively.

Hence $A G\left(Z_{\rho q}\right)=K_{(p-1)} \cup K_{(q-1)} \cup K_{p q-p-q+1}$.

$3.12 \mathrm{C}$ - program to find the components of $A G\left(Z_{n}\right): A c$-programming is prepared to find the components of $A G\left(Z_{n}\right)$ for a given positive integer $n$.

Example:

Enter ' $n$ ' value: 50

ORBIT 1: $\{1,3,7,9,11,13,17,19$,

$21,23,27,29,31,33,37,39,41,43$

$47,49\}$.

No. of elements is : 20

ORBIT 2: $\{2,4,6,8,12,14,16,18$,

$22,24,26,28,32,34,36,38,42,44$, $46,48\}$.

No. of elements is : 20

ORBIT 5: $\{5, \quad 15,35,45\}$.

No. of elements is : 4

ORBIT 10: $\{10,20,30,40\}$.

No. of elements is : 4

ORBIT 25: $\{25\}$. 
No. of elements is : 1

FINAL SET is: $\{1,4,4,20,20\}=49$.

Thus $A G\left(Z_{50}\right)=K_{1} \cup K_{4} \cup K_{4} \cup K_{20} \cup K_{20}$.

\section{References}

1. I. Beck, Coloring of Commutative Rings, J. AJgebra 116 (1988), 208-226.

2. D.D. Anderson and M. Nseer, Beck's Coloring of a Commutative Ring, J. Algebra 159 (1993), 500-514.

3. V.K. Bhat and Ravi Raina, Neeraj Nehro, A Note on Zero Divisor Graph Over.

4. I.N. Herstein, Topics in Algebra, Wiley Eastern Limited.

5. F. Harary, Graph Theory, Addison-Wesley, Reading, MA, 1972. 